Rechtsmedizin $2022 \cdot 32: 277-281$

https://doi.org/10.1007/s00194-021-00531-3

Angenommen: 6. August 2021

Online publiziert: 15 . September 2021

(c) Der/die Autor(en) 2021

\section{Auftragsmord und Anstiftung zum Mord als Varianten des Intimizids}

\author{
E. Dietz · J. Schädler · K. Püschel · B. Ondruschka \\ Institut für Rechtsmedizin Hamburg, Universitätsklinikum Hamburg-Eppendorf, Hamburg, Deutschland
}

\title{
Zusammenfassung
}

Dargestellt wird der Fall eines Intimizids, bei dem sich ein Ehemann durch Anstiftung zum Mord seiner Ehefrau entledigen wollte. Auftragsmorde bzw. Anstiftungen zum Mord innerhalb von Paarbeziehungen stellen ein Ausnahmegeschehen dar. Hier sollen die Facetten einer umfangreichen kriminalistischen Fallbearbeitung dargestellt werden, die neben rechtsmedizinischen Erkenntnissen auch wesentliche psychiatrische, psychologische und kriminalistische Aspekte der Tatbegehung bzw. -anstiftung beleuchtet.

\section{Schlüsselwörter}

Beziehungsgewalt · Ehegattinnenmord · Täter-Opfer-Beziehung $\cdot$ Mordmotive $\cdot$ Stichkanalrekonstruktion

\section{Hintergrund}

Der klassischen Auftragsmord (",contract murder") findet sich u.a. definiert als Ermordung eines Menschen durch einen sog. Auftrags-/Berufsmörder. Dies ist meistens eine außenstehende, unbeteiligte, dritte Person ohne erkennbare Vorbeziehung zum Opfer. Die Ausführung eines Auftragmords erfolgt regelhaft gegen eine Entlohnung. Diese wird vom Auftraggeber bereitgestellt, der (häufig) eine Vorbeziehung zum Opfer hat [1]. Dem klassischen Auftragsmord ist die Instrumentalisierung einer Person als Täter(in) zu Selbstzwecken gegenüberzustellen. Hierfür gibt es verschiedenste Motivationslagen, die keineswegs nur der Generierung eines eigenen wirtschaftlichen Benefits dienen, sondern mitunter auch Tötungen einschließen können. Häufig besteht ein direktes persönliches, sexuelles und/oder emotionales Abhängigkeitsverhältnis zum Tatinitiator.

Beide Varianten finden sich u.a. auch im Kontext von Partnertötungen (Intimiziden) beschrieben. Diese beiden Konstellationen sind wiederum von der klassischen Partnertötung (einschließlich der Affektdelikte) abzugrenzen, bei der keine weiteren Personen in den Tötungsakt bzw. das Tötungsvorhaben involviert sind. Den verschiedenen Konstellationen gemeinsam ist die gezielte Tötung des Partners, die gewöhnlich - mit Ausnahme von klassischen Affekttaten im Zusammenhang mit Vorplanung und Manipulation in Erscheinung tritt.

Erfolgt die Tötung durch eine dritte Person, d. h. nicht durch den Partner selbst, so ist bei den die (Tat-)Handlungen ausführenden Personen zwischen professionellen („professional murderer, killer") und plötzlichen („sudden murderer ${ }^{\prime \prime}$ ) Mördern zu differenzieren. Der plötzliche Mörder unterscheidet sich gemeinhin vom professionellen Mörder dadurch, dass er plötzlich, absichtlich, jedoch ohne eigene Intention einen anderen Menschen tötet oder einen ernsthaften Versuch hierzu unternimmt, ohne je zuvor getötet zu haben oder bisher in vergleichbarer Weise (beispielsweise durch schwer psychotische, aggressive und antisoziale Aktionen) in Erscheinung getreten gewesen zu sein (entsprechend einer sog. Täter-/Mörderwerdung) [2, 3].

Die Motive des Intimizids entsprechen denen eines Mords und umfassen in Abhängigkeit vom soziokulturellen Hintergrund u.a. verletztes Ehrgefühl, 


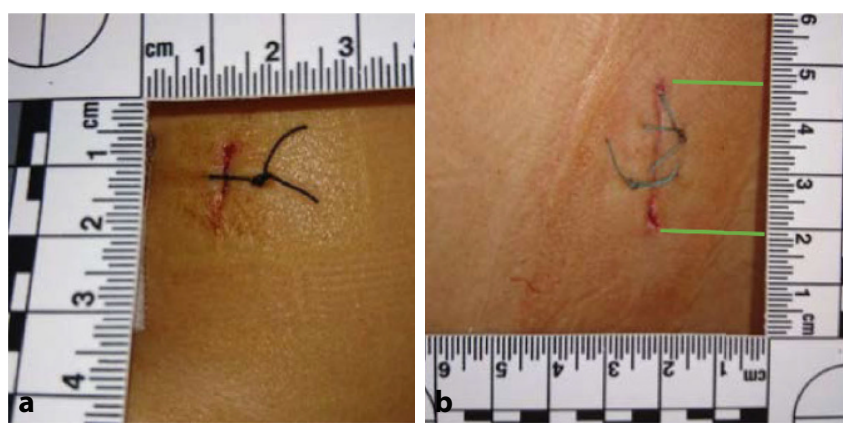

Abb. 1 \ Äußerlich feststellbare Verletzungen: a Unterhalb des linken unteren Brustansatzes eine längs gestellte, etwa $1,5 \mathrm{~cm}$ lange, glattrandige, mittels chirurgischer Einzelknopfnaht adaptierend verschlossene Hautläsion. b Zwischen dem rechten Schulterblatt und der Wirbelsäule eine in Längsrichtung leicht schräg gestellte, etwa $3 \mathrm{~cm}$ lange, glattrandige, mittels chirurgischen Einzelknopfnähten adaptierend verschlossene Hautläsion
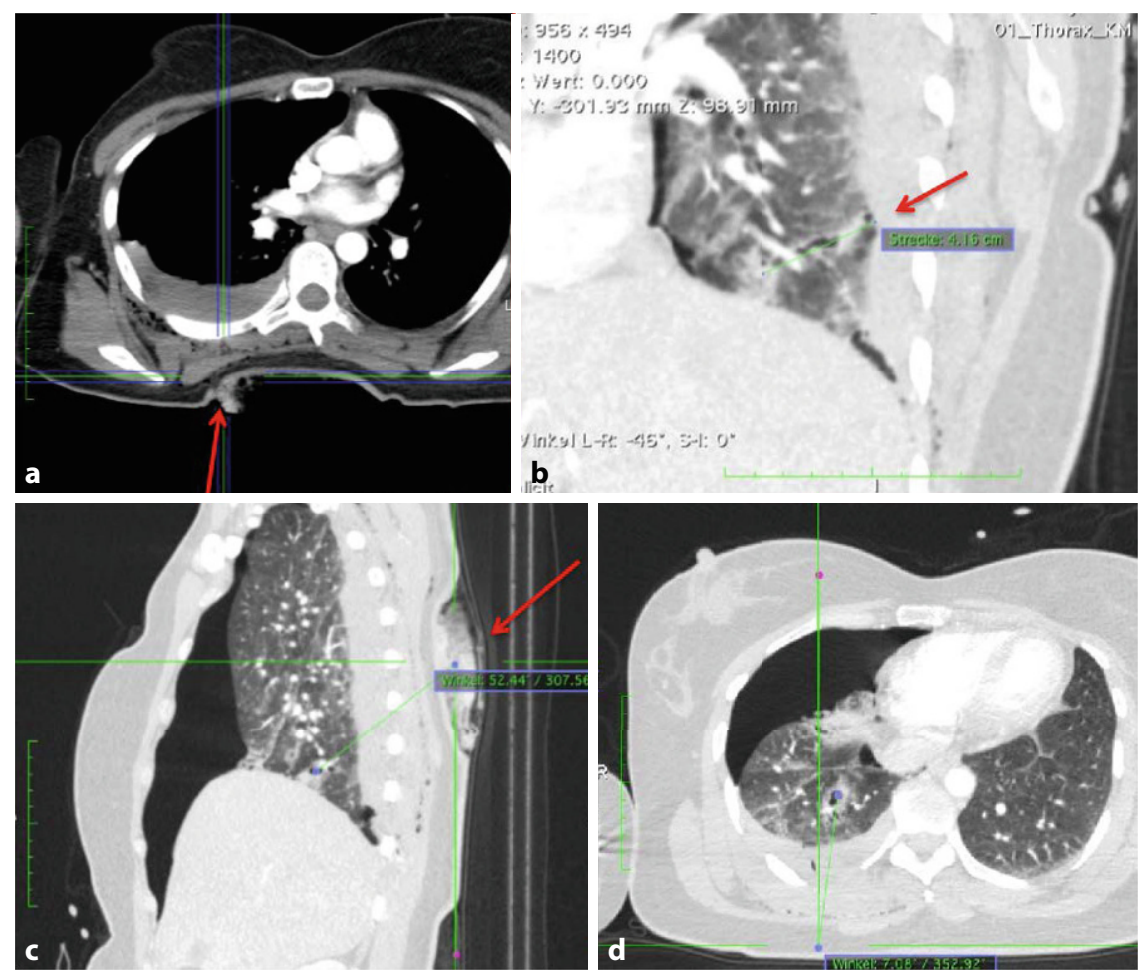

Abb. 2 \ Stichkanalrekonstruktion anhand der klinischen CT-Daten: a Axiale Schnittebene: Stichwunde zwischen rechtem Schulterblatt und Wirbelsäule, leicht schräg absteigend. b Schräge Schnittebene: Mindestlänge des Lungenstichkanals im rechten Unterlappen mit Darstellung der umbluteten Gasstraße. c Pfeilebene: Stichkanal deutlich absteigend, Bezugspunkte: Hautperforation und Stichkanalende in rechtem Lungenunterlappen. $\mathbf{d}$ Axiale Schnittebene: Stichkanal mit Anfangs- und Endpunkt bei direkter Verbindung der Hautoberfläche mit dem Lungenstichende. Die roten Pfeile markieren jeweils den rekonstruierten Stichkanalverlauf

enttäuschte Liebe, Kränkung/Zorn, Rache, Hass, Emanzipations- und Befreiungsausbruch, Geldgier und Bereicherung [4, 5]. Die Fallzahlen der Tötungen innerhalb von partnerschaftlichen Beziehungen belaufen sich konstant auf etwa $20 \%$ aller Tötungsdelikte [6]. Partnertötungen sind im Vergleich zu nicht tödlich endenden Viktimisierungen innerhalb von Paarbe- ziehungen eher seltene Ereignisse [7]. Den Täter-Opfer-Konstellationen ist zu entnehmen, dass Intimizide überwiegend von Männern begangen werden; die Opfer sind überwiegend weiblich [5, 6]. Klassische Auftragsmorde oder die Instrumentalisierung einer dritten Person zur Mordausführung innerhalb von Partnerschaften sind - zumindest im
Hamburger Einzugsbereich und in der einschlägigen Literatur - eine Seltenheit. Hinsichtlich der Mordmittel und der Tatausführungen i. Allg. können solche vom "männlichen" und solche vom "weiblichen" Typ differenziert werden; ähnlich der geschlechtsspezifischen Wahl von "harten" und "weichen" Suizidmethoden. Diese Arten der Mordmittel bzw. Tatausführung sind auch auf Auftragsmorde übertragbar [8-10].

\section{Falldarstellung}

\section{Ausgangssituation}

Bei der klinisch-rechtsmedizinischen Untersuchung eines schwer verletzten Gewaltopfers in einem Krankenhaus, einen Tag nach dem Vorfall, gab die Geschädigte an, dass sie in ihrer Wohnung unvermittelt von einer ihr unbekannten Frau angegriffen worden sei. Die Angreiferin habe ihr mit einem in der rechten Hand gehaltenen Küchenmesser mit einseitig schneidender Klinge in die linke Brustkorbhälfte gestochen. Daraufhin habe sie sich aktiv zur Wehr gesetzt. Jetzt habe die Frau ihr mit einer in der linken Hand gehaltenen Schusswaffe (mutmaßlich Pistole) mehrmals auf den Kopf geschlagen. Die Geschädigte habe die Angreiferin angefleht aufzuhören. Die Angreiferin habe in Aussicht gestellt zu gehen, wenn die Geschädigte die Arme über den Kopf halte und sich umdrehe. Nun habe die Angreiferin ihr in den Rücken gestochen und mit der "Pistole" auf das bereits in ihrem Rücken steckende Messer geschlagen. Der alarmierte Rettungsdienst habe die Geschädigte kurze Zeit später schwer verletzt in eine Klinik verbracht.

\section{Verletzungsmuster/Rekonstruktion}

Klinisch wurden eine blande Stichverletzung am linken Oberbauch ohne Eröffnung der Bauchhöhle (ब Abb. 1a) sowie eine tiefe Stichverletzung im rechten oberen Rückendrittel zwischen Schulterblatt und Wirbelsäule mit Eröffnung des Brustkorbs und Ausbildung eines Hämatopneumothoraxes rechts festgestellt (- Abb. 1b). Letztere bedurfte einer sofortigen notfallmedizinischen Intervention. Rechtsmedizinischerseits stellten sich die Verletzungen als glattrandige und chi- 
rurgisch versorgte Hautdurchtrennungen dar (• Abb. 1). Zusätzlich fanden sich an den oberen Extremitäten, insbesondere an den Handinnen- und Handaußenseiten, charakteristische Verletzungen, die als aktive und passive Abwehrverletzungen interpretiert wurden. Die behaarte Kopfhaut zeigte sich unverletzt.

Eine zusätzlich durchgeführte Stichkanalrekonstruktion (einschließlich der Fragestellung auf Hinweise eines Nachstechens) anhand der klinischen CT-Bilder (- Abb. 2) ergab folgende Befunde:

1. Rückwärtige Stichverletzung zwischen rechtem Schulterblatt und Wirbelsäule, korrespondierend mit einer Mindeststichkanallänge von ca. $11 \mathrm{~cm}$. Der Stichkanal deutlich absteigend (ca. 50-55 Grad) und gering von hinten-außen nach vorne-innen gerichtet. Darstellbare Mindestlänge des Lungenstichkanals im rechten Lungenunterlappen ca. 4,2 cm. Keine radiologischen Hinweise auf eine Mehrfachkanalbildung.

2. Stichverletzung unterhalb der linken Brust. Absteigender, ca. $3 \mathrm{~cm}$ tiefer und bis zum Brustbeinknorpelgewebe (Höhe des Ansatzes der 8. Rippe) reichender, sich als Gewebeeinblutung darstellender Stichkanal.

\section{Ermittlungsergebnisse}

Durch das Landeskriminalamt konnten der Ehemann und eine gute und langjährige Bekannte des Ehemannes als Tatverdächtige ermittelt werden. Im strafprozessualen Verfahren legte der Ehemann ein Geständnis ab und gab an, nach zuvor zwei missglückten Auftragsmordversuchen seine langjährig Bekannte und Mitangeklagte zur Tatausführung verleitet („,instrumentalisiert") zu haben. Die Mitangeklagte führt an, dass sie das Opfer bereits wiederholt verfolgt habe, jedoch ohne eine geeignete Möglichkeit erlangen zu können, das Tatvorhaben umzusetzen. Daraufhin sei der Plan entstanden, die Geschädigte durch Messerstiche zu Hause zu töten, welches letztlich erneut scheiterte. Die gute Bekannte des Ehemannes gab an, nicht aus wirtschaftlichen Gründen gehandelt zu haben, sondern vielmehr aus einem Wechselbad der Gefühle mit evozierter emotionaler Reaktion (sie sei darum gebeten worden und habe es „nie geschafft", nein zu sagen).

\section{Motiv}

Das Motiv sei nach Angaben des Angeklagten zunächst von dem Wunsch nach der Beendigung seiner unglücklichen Beziehung geleitet worden. Darüber hinaus habe er Sorge gehabt, bei einer Scheidung das Sorgerecht für den gemeinsamen Sohn zu verlieren. Es stellte sich heraus, dass der Mann aufgrund seiner Lebensführung hoch verschuldet war. Durch Initiierung des Todes der Geschädigten habe sich der Angeklagte erhofft, die Todesfallleistungen aus der Lebensversicherung seiner Frau zu erlangen. Dieses Gewinnbestreben war letztlich das führende Tatmotiv. Die Tötung seiner Frau nicht selbst durchzuführen, sei dem Gedanken entwachsen, sich hierdurch weniger schuldig zu machen und überhaupt die Möglichkeit aufrechtzuerhalten, die Todesfallleistung der Lebensversicherung seiner Frau erlangen zu können.

\section{Urteil}

Die Anstiftung zum Mord wurde juristisch schwerer gewichtet als die eigentliche Ausführung der versuchten Tötung. Der Ehemann erhielt eine Haftstrafe von 8 Jahren und 6 Monaten wegen versuchten Mordes in Tateinheit mit gefährlicher Körperverletzung. Er verwirklichte zwei Mordmerkmale: Er handelte aus Habgier, und die Tötung sollte es ihm ermöglichen, weitere Straftaten zu begehen. Die Todesfallleistung der Lebensversicherung hätte er nur erhalten, wenn er der Versicherung angegeben hätte, nicht an der Tötung seiner Frau beteiligt gewesen zu sein. Die Mitangeklagte erhielt eine Haftstrafe von 4 Jahren und 8 Monaten wegen versuchten Totschlages mit gefährlicher (gemeinschaftlichen) Körperverletzung. Mordmerkmale waren in ihrem Fall nicht erfüllt. Aufgrund einer nicht näher beschriebenen psychischen Erkrankung lag bei ihr eine verminderte Schuldfähigkeit vor. Zudem fand ein Täter-Opfer-Ausgleich statt. Des Weiteren legte sie ein umfangreiches Geständnis $a b$, welches die vollständige Aufdeckung der Tat erst ermöglichte. Diese drei Punkte führten letztlich zu einer Strafminderung bei der Angeklagten.

\section{Diskussion der Erkenntnisse und Schlussfolgerungen}

Der Fall zeigt deutlich, dass es sich bei Partnertötungen keinesfalls nur um das klassische Bild der Affektdelikte handelt. Eine besondere Belastung des Täters bzw. des Tatinitiators im Vorfeld der Tat muss nicht erkennbar sein. Außer- und innerpartnerschaftliche Gewalt, im Sinne eines psychotischen, aggressiven und/oder zur Gewalt neigenden Persönlichkeitsprofils, muss nicht vorausgehen. Die Anstiftung zum Mord oder die bezahlte Auftragstötung stellen aus Tätersicht profane Mittel zur Verwirklichung der Leitidee des Intimizids dar. Möglicherweise sieht der Auftraggeber in der Fremdtötung durch einen helfenden Dritten selbst - zumindest gedanklich - kein unmittelbares Unrecht verwirklicht, d.h., er rechnet der ausführenden Person die letztliche Tötung zu. Auch kann in der Handlung durch einen Dritten eine Minimierung des Strafverfolgungsrisikos vermutet werden, beispielsweise durch eine Alibiverschaffung oder Verschleierung der Tat.

Die Instrumentalisierung von Mitmenschen zu Selbstzwecken, einschließlich der Tötung einer anderen Person (ausgenommen professioneller Auftragsmörder), scheint insbesondere dann möglich zu sein, wenn u.a. die zu instrumentalisierende Person in einem Abhängigkeitsverhältnis (ungeachtet der Art und Weise) zum Auftraggeber steht oder eine labile, psychotisch-antisoziale und/oder aggressive Primärpersönlichkeitsstruktur aufweist [11, 12].

Im Gegensatz zum klassischen Auftragsmord, bei dem die Tatausführung durch einen professionellen Berufskiller ("contract killer“ oder ",hitman") erfolgt, ist das Beziehungsgeflecht zwischen Täter, Opfer und "Auftraggeber" im Falle einer Anstiftung zum Mord unter Bedienung eines Laienmörders oder eines noch nie zuvor mordenden Menschen in der Regel einfacher aufzudecken. Die vordeliktische Täter-Opfer- bzw. TäterAnstifter-Opfer-Beziehung erhöht sonach das Strafverfolgungsrisiko, einschließlich 
des Risikos einer schnellen Tataufklärung und Verurteilung [13].

Der geschilderte Fallbericht lässt Tätermerkmale vom weiblichen Typ erkennen. Wird den Ausführungen des Opfers zum Geschehensablauf gefolgt, so könnte der Umstand des ersten missglückten Tötungsversuches durch einen Bruststich u.a. auf Hemmungen beim Töten von Angesicht zu Angesicht und das Erfordernis eines direkten und aktiven Vorgehens zurückzuführen sein. Die aktive Gegenwehr des Opfers und die daraus resultierende Kampfhandlung, evtl. verbunden mit einer angenommenen bzw. befürchteten körperlichen Unterlegenheit vonseiten der Täterin, beeinflusste offensichtlich den weiteren Geschehensablauf. Im gegenständlichen Fall lag jedoch nur eine schwache Fundierung der Motivlage der Täterin - im Sinne fehlender reaktiver (beispielsweise impulsiv aus der Wut heraus) und/oder instrumenteller (beispielsweise aus berechnender Motivlage heraus) Aggressionen vor.

Dies scheint sich auch im Ergebnis des Tatablaufs zu bestätigen. So wurde das Opfer unter Vorspiegelung eines falschen Versprechens dazu gebracht, die aktive Gegenwehr zu unterlassen und sich schutzlos von der Täterin abzuwenden, wobei diese zeitgleich zu einem weiteren Stich (von hinten oben) ansetzte und die möglicherweise aus Hemmungen bzw. fehlender Aggression heraus fehlende Stichkraft mit einem Nachschlagen auf das angesetzte Messer (Hammerwirkung) kombinierte.

\section{Fazit für die Praxis}

Mord- und Totschlagsdelikte ereignen sich in vielen Fällen im sozialen Nahfeld. Der Täter bzw. die Täterin stammen nicht selten aus der Familie oder dem engen Freundeskreis. Auftragsmorde und Anstiftungen zum Mord sind profane Mittel des Gattenmordes, wenngleich klassische Affektdelikte die Intimizide dominieren. Das Verletzungsbild eines Opfers, einschließlich der Tatortspuren, erlaubt es teilweise, das Tatgeschehen zu rekonstruieren oder Aussagen zum Motiv und zum Tätertyp zu treffen. Täter-Opfer-Beziehungen und die Tat-/Täterzusammenhänge mit sozialer und räumlicher Nähe zum Opfer erhöhen die Chance einer schnellen Tataufklärung und Verurteilung.

\section{Contract murder and incitement to murder as variants of intimate partner homicide}

A case of intimate partner homicide is presented, in which a husband wanted to get rid of his wife by incitement to murder. Contract murders and incitement to murder within a partner relationship are exceptional events. Various facets, such as forensic pathologic, psychological and criminalistic aspects in cases of contract murder or incitement to murder are presented in addition to extensive criminal case processing of this special case.

\section{Keywords}

Domestic violence $\cdot$ Intimate murder $\cdot$ Victim-offender relationship $\cdot$ Murder motive $\cdot$ Stab canal reconstruction

\section{Korrespondenzadresse}

\section{Dr. med. E. Dietz}

Institut für Rechtsmedizin Hamburg, Universitätsklinikum Hamburg-Eppendorf Butenfeld 34, 22529 Hamburg, Deutschland e.dietz@uke.de

Funding. Open Access funding enabled and organized by Projekt DEAL.

\section{Einhaltung ethischer Richtlinien}

Interessenkonflikt. E. Dietz, J. Schädler, K. Püschel und B. Ondruschka geben an, dass kein Interessenkonflikt besteht.

Die Untersuchungen erfolgten unter Einhaltung der Vorgaben der Zentralen Ethikkommission der Bundesärztekammer.

Open Access. Dieser Artikel wird unter der Creative Commons Namensnennung 4.0 International Lizenz veröffentlicht, welche die Nutzung, Vervielfältigung, Bearbeitung, Verbreitung und Wiedergabe in jeglichem Medium und Format erlaubt, sofern Sie den/die ursprünglichen Autor(en) und die Quelle ordnungsgemäß nennen, einen Link zur Creative Commons Lizenz beifügen und angeben, ob Änderungen vorgenommen wurden.

Die in diesem Artikel enthaltenen Bilder und sonstiges Drittmaterial unterliegen ebenfalls der genannten Creative Commons Lizenz, sofern sich aus der Abbildungslegende nichts anderes ergibt. Sofern das betreffende Material nicht unter der genannten Creative Commons Lizenz steht und die betreffende Handlung nicht nach gesetzlichen Vorschriften erlaubt ist, ist für die oben aufgeführten Weiterverwendungen des Materials die Einwilligung des jeweiligen Rechteinhabers einzuholen.

Weitere Details zur Lizenz entnehmen Sie bitte der Lizenzinformation auf http://creativecommons.org/ licenses/by/4.0/deed.de.

\section{Literatur}

1. Mouzos J, Venditto J (2004) Contract killings in Australia. Research and public policy series, Bd. 53. Australian Institute of Criminology, Canberra, S 1-82

2. Weiss JMA, Lamberti JW, Blackman N (1960) The sudden murderer: a comparative analysis. AMA Arch Gen Psychiatry 2(6):669-678. https://doi.org/ 10.1001/archpsyc.1960.03590120077009

3. Ruotolo AK (1968) Dynamics of sudden murder. Am J Psychoanal 28(2):162-176. https://doi.org/ 10.1007/BF01873635

4. Kröber HL (2015) Die Mordmerkmale aus forensisch-psychiatrischer Sicht. Forens Psychiatr Psychol Kriminol 9(4):251-257. https://doi.org/10. 1007/s11757-015-0340-5

5. Leygraf N (2015) Tötungsdelikte in und nach intimen Beziehungen. Forens Psychiatr Psychol Kriminol 9(4):211-219. https://doi.org/10.1007/ s11757-015-0342-3

6. Mützel $E$, Auberlen-Pacholke A, Lindemaier $G$ et al (2014) Intimizid in Bayern in den Jahren 2004-2007. Rechtsmedizin 24(6):494-501. https://doi.org/10.1007/s00194-014-0982-1

7. Gemünden J (2003) Gewalt im Hell- und Dunkelfeld. Zur empirischen Relevanz der Gewalt gegen Männer. In: Lamnek S, Boatcă M (Hrsg) Geschlecht - Gewalt-Gesellschaft. Leske + Budrich, Opladen, S333

8. Harbort S (2009) Interview mit Klaus Pokatzky zum Start der ARD-Reihe „Wenn Frauen morden". https://www.deutschlandfunkkultur.de/frauentoeten-eben-ganz-anders.954.de.html?dram: article_id=143942.Zugegriffen:07.08.2019

9. Haltenhof H (2004) Suizidalität. In: Machleidt W, Bauer M, Lamprecht F et al (Hrsg) Psychiatrie, Psychosomatik und Psychotherapie, 7. Aufl. Thieme, Stuttgart, New York, S 241-242

10. Uhl K (2003) Die Gewaltverbrecherin im kriminologischen und literarischen Diskurs des frühen 20. Jahrhunderts. In: Hilbig A, Kajatin C, Miethe I (Hrsg) Frauen und Gewalt:Interdisziplinäre Untersuchungen zu geschlechtsgebundener Gewalt in Theorie und Praxis. Königshausen \& Neumann, Würzburg, S91

11. Hoff P, Sass H (2010) Psychopathologische Grundlagen der forensischen Psychiatrie. In: Kröber HL, Dölling D, Leygraf $\mathrm{N}$ et al (Hrsg) Psychopathologische Grundlagen und Praxis der Forensischen Psychiatrie im Strafrecht. Handbuch der forensischen Psychiatrie, Bd. 2. Springer, Berlin, Heidelberg, New York, S 1-144 


\section{Buchbesprechung}

12. Dulz B, Briken P, Kernberg OF et al (Hrsg) (2017) Handbuch der Antisozialen Persönlichkeitsstörung. Schattauer, Stuttgart, S259-279

13. Maclntyre D, Wilson D, Yardley E et al (2014) The British hitman: 1974-2013. Howard J Crim Justice 53(4):325-340. https://doi.org/10.1111/ hojo. 12063

B. Madea, J. Prangenberg, J. Ulbricht,

E. Doberentz

Feststellung der Todesursache

Berlin: Lehmanns Media 2022, 1. Aufl., 235 S., (ISBN: 978-3-96543283-3), 14,95 EUR

Mit dem Buch machen es sich die Autoren zur Aufgabe, ihren Lesern die Problematik der Todesursachenfindung und der Erstellung einer plausiblen Todesursachenkaskade näher zu bringen. Neben einem kurzen Exkurs in die Historie werden Grundlagen zu Begrifflichkeiten und Klassifizierungen vermittelt. Ein zweiter Block widmet sich dem eigentlichen Thema. Tabellarisch sowie anhand zahlreicher Abbildungen werden hierzu Leitfäden und Hilfsmittel präsentiert. Interesse und Aufmerksamkeit wecken die anschaulich bebilderten Fallbeispiele, gefolgt von weiteren Exempeln zu Todesursachenkaskaden, mithilfe derer der Fokus auf die praktische Umsetzung gelegt wird. Darüber hinaus werden jedoch auch die Limitationen der ärztlichen Leichenschau bei der endgültigen Feststellung der Todesursache aufgezeigt. Mögliche Fehlerquellen werden klar benannt und vermitteln damit ein Verständnis für die Verantwortung der mit der Todesursachendiagnostik betrauten Ärzt:innen. Die letzten Kapitel des Buches enthalten Beispiele von sowohl plausiblen als auch nicht plausiblen Todesursachenkaskaden. Hierbei werden in verschiedensten Konstellationen mögliche Kausalitätsketten tabellarisch dargestellt. Ihrem Anspruch, möglichst vielen Krankheiten und die unterschiedlichsten Settings aufzuführen, kommen die Autoren damit durchaus nach. Fehlerquellen bei nicht plausiblen Kaskaden werden zwar benannt, könnten dem Leser jedoch anhand korrigierter Versionen noch präziser vermittelt werden. Insgesamt ist der Inhalt jedoch gut verständlich und wird dem Bestreben, häufige Fragen zur Erstellung einer sinnvollen Todesursachenkaskade mit realen Beispielen zu beantworten, gerecht. Das Buch richtet sich nicht nur an Studierende der Medizin, sondern ist auch ein praxisorientierter Leitfaden für klinisch tätige Ärzt:innen. 